PUBLISHER CORRECTION

\title{
Correction to: Two generalized Tricomi equations
}

\section{Fabio Paronetto ${ }^{1}$}

Published online: 31 August 2021

(c) Springer-Verlag GmbH Germany, part of Springer Nature 2021

\section{Calc. Var. (2021) 60:84 https://doi.org/10.1007/s00526-021-01970-2}

In the published article, fiagure 1 caption and some lines in the section 4.2 and 4.3 were published incorrectly due to an error on the publisher's side. The correct caption and lines are given below.

Caption for Figure 1: Two possible examples in which $\mathcal{R}^{\prime} \leq 0$.

2. Modifying the initial datum $\eta$ - Indeed ...

3. Boundedness for the solutions $u_{\epsilon}$ - Denote by $\ldots$

4. Taking the limit on the boundary conditions concerning $u_{\epsilon}$ - In this step...

6. Passage to the limit on the boundary conditions concerning $u_{\epsilon}$ - The last ...

8. Convergence of the all family $\left\{u_{\epsilon}\right\}_{\epsilon>0}$ - In ...

3. Boundedness for the solutions $u_{\epsilon}$ - One ...

Publisher's Note Springer Nature remains neutral with regard to jurisdictional claims in published maps and institutional affiliations.

The original article can be found online at https://doi.org/10.1007/s00526-021-01970-2.

Fabio Paronetto

fabio.paronetto@unipd.it

1 Dipartimento Di Matematica Tullio Levi Civita, Università Degli Studi Di Padova, Via Trieste 63, Padova, Italy 